DÍEZ RIPOLLÉS, José Luis; GARCÍA, Elisa: "RIMES: Un instrumento de comparación

de políticas criminales nacionales desde la exclusión social"

Polít. Crim. Vol. 15, No 30 (Diciembre 2020), Art. 6, pp. 670-693

[http://politcrim.com/wp-content/uploads/2020/12/Vol15N30A6.pdf]

\title{
RIMES: Un instrumento de comparación de políticas criminales nacionales desde la exclusión social ${ }^{*}$
}

\section{RIMES: An Instrument to Compare National Criminal Justice Policies from the Social Exclusion Dimension}

\author{
José Luis Díez Ripollés \\ Catedrático Derecho Penal \\ Universidad de Málaga (España) \\ ripolles@uma.es \\ Elisa García España \\ Profesora Titular de Derecho Penal \\ Universidad de Málaga \\ Elisa@uma.es
}

\section{Resumen}

El artículo presenta una herramienta válida para comparar sistemas de control penal nacionales desde la perspectiva de la exclusión social que producen sobre aquellos colectivos que entran en conflicto con la ley penal. Se exponen los motivos por los que se ha escogido la dimensión de la exclusión social como criterio de comparación de políticas criminales nacionales, en lugar de otros criterios más usuales. A continuación, se explican los objetivos específicos, metodología, elaboración y configuración final del instrumento, que se llama RIMES. El instrumento, que consta de 39 indicadores consistentes en reglas y prácticas punitivas reales que abarcan nueve grandes áreas de intervención penal, está en condiciones de medir de forma comprensiva la exclusión social generada sobre sospechosos, procesados, condenados y excondenados por los diversos sistemas de control penal de los países occidentales desarrollados. De este modo, posibilita la realización de comparaciones entre los diversos países. El instrumento ha sido sometido a un doble proceso de validación de contenidos por juicio de expertos en el que han participado cerca de 100 reconocidos expertos de 18 países del mundo occidental desarrollado. Se pretende en último término disponer de una herramienta fiable para interpretar la política criminal contemporánea y para fomentar decisiones político-criminales fundamentadas.

Palabras clave: Política criminal comparada, instrumento de medición comparada, exclusión social, instrumento RIMES.

\footnotetext{
* Este artículo se realiza en el marco del Proyecto I+D de Generación de conocimiento denominado "La exclusión social como criterio de comparación político-criminal: Aplicación del instrumento RIMES" (APRIMES) (PGC2018-096073-B-I00) de las investigadoras principales Elisa García España y Anabel Cerezo. Financiado por el Programa estatal de generación de conocimiento y fortalecimiento científico y tecnológico del sistema de I+D+I.
} 


\title{
Polít. Crim. Vol. 15, No 30 (Diciembre 2020), Art. 6, pp. 670-693 [http://politcrim.com/wp-content/uploads/2020/12/Vol15N30A6.pdf]
}

\begin{abstract}
This paper describes a validated tool for comparing national crime control systems in accordance to the social exclusion they generate on those groups prone to enter into conflict with the criminal law. First, we first explain why we chose social exclusion as guiding principle for comparing national criminal policies, instead of other more usual criteria like punitiveness. Then, we describe objectives, methodology, development, and the final configuration of the comparative instrument called RIMES. The instrument comprises 39 indicators made up of current punitive rules and practices concerning nine relevant fields of penal intervention. RIMES measures in a comprehensive manner the social exclusion caused on suspects, defendants, offenders and ex-offenders by crime control systems of different Western industrialized countries, in a way that it makes possible to proceed to comparisons across different countries. The tool was submitted to a double process of validation by inter-judge agreement, where about 100 well-known international experts from 18 Western developed countries took part. Our last goal is to make available a reliable instrument for interpreting current criminal justice policies and fostering grounded criminal policy decisions.
\end{abstract}

Keywords: Comparative criminal policy, instrument for comparative measurement, social exclusion, RIMES instrument.

\section{Introducción}

La actual política criminal comparada muestra una fuerte tendencia a contrastar los diferentes sistemas de control penal nacionales en función de su mayor o menor rigor punitivo. Este enfoque analítico parte de un presupuesto indiscutido: todo sistema de control penal debería tener como uno de sus objetivos primordiales asegurar que quienes entran en conflicto con la ley penal sufran una aflicción moderada como consecuencia de su comportamiento delictivo o sospechoso. ${ }^{1}$

Sin embargo, utilizar como criterio rector de los análisis de política criminal comparada el grado de moderación punitiva alcanzado por los respectivos sistemas de control penal presenta un conjunto de defectos teóricos y metodológicos.

Entre los primeros, cabe destacar que el objetivo de la moderación punitiva se inserta en un contexto ideológico pobre ${ }^{2}$ : constituye una aproximación humanitaria a la política criminal. Esta obtendría reconocimiento en cuanto garantice que la dureza de las reacciones penales no supera cierto límite, con independencia de la corrección del conjunto de objetivos político-criminales perseguidos y del modelo político-criminal adoptado para conseguirlos.

Entre los defectos metodológicos resalta el hecho de que, aun aceptado el enfoque, utiliza indicadores de moderación punitiva excesivamente limitados. En realidad, casi todas las comparaciones giran básicamente en torno a la tasa de encarcelamiento por cada 100.000 habitantes. No se niegan las virtudes de este indicador: es fácilmente accesible a partir de

\footnotetext{
${ }^{1}$ HINDS (2005), pp. 346; CAVADINO Y DIGNAN (2006), pp. 380; DOWNES Y HANSEN (2006), passim; TONRY (2007), passim; LAPPI-SEPPÄLÄ, (2008), pp. 313-387; LARRAURI (2009), pp. 1-22, entre otros.

${ }^{2}$ Es pobre exclusivamente en el sentido de que con solo un enfoque humanitario no se puede abordar estrategias político-criminales analíticas e intervencionistas suficientemente complejas.
} 
fuentes fiables, se centra en una de las sanciones más duras del catálogo punitivo, y ejemplifica bien el conjunto de políticas y prácticas punitivas de un sistema penal. Sin embargo, concentrar la evaluación del rigor penal en el uso de la pena de prisión margina otros indicadores fuertemente expresivos, como, por ejemplo, número de procedimientos penales condenatorios, duración media de las diversas penas, entre otros. Es más, la intensidad del uso de la prisión depende también del número de ingresos, de la estancia media, de internamientos no penitenciarios, etc. ${ }^{3}$

La propuesta cuyo desarrollo se va a describir en este artículo aboga por realizar la comparación político-criminal internacional a partir de un enfoque más rico y comprensivo, y empleando una pluralidad organizada de indicadores. Lo cual no quiere decir que el objetivo de la moderación punitiva o el indicador para medirla deban ser descartados.

\section{Modelo teórico}

El modelo teórico adoptado para diseñar la presente investigación se funda en el defendido por Díez-Ripollés, y escoge como elemento de comparación la medida en que los correspondientes sistemas de control penal minimizan la exclusión social de los que entran en conflicto con la ley penal.

Es importante señalar que el modelo teórico aludido no va referido a los efectos que el sistema de control penal produce sobre el conjunto de la población. Queda confinado a las personas y colectivos que son objeto prioritario de los órganos de prevención y persecución penales, a saber, quienes han estado, están o son proclives a estar sometidos directamente al control penal en su calidad de excondenados, condenados, procesados o sospechosos.

La búsqueda de la exclusión o de la inclusión social de las personas proclives a chocar con la ley penal refleja dos aproximaciones contrapuestas al objetivo político-criminal de prevenir la delincuencia. El enfoque socialmente excluyente aspira sustancialmente a lograr la inocuización de los colectivos señalados, lo que implica asegurar que el excondenado, condenado, procesado o sospechoso se encuentre, tras su contacto con las instituciones penales, en unas condiciones individuales y sociales en las que le resulte más difícil infringir la ley o evitar ser descubierto. Por el contrario, un enfoque socialmente incluyente busca ante todo la reinserción social de los citados colectivos, de modo que el excondenado, condenado, procesado o sospechoso se encuentre, tras su contacto con las instituciones penales, en iguales o mejores condiciones individuales y sociales para desarrollar voluntariamente una vida conforme con la ley.

En consecuencia, los diferentes sistemas de control penal nacionales habrán de evaluarse según el grado en que sus modelos de intervención penal se acerquen a uno de los dos enfoques. Nuestra investigación, más modestamente, pretende medir el mayor o menor

\footnotetext{
3 BALVIG (2004), pp. 167-187; BRODEUR (2007), pp. 49-91; WEBSTER y DOOB (2007), passim; ROCHÉ (2007), passim; SNACKEN (2007), passim; ZAFFARONI (2007), pp. 221; PRATT (2008), passim; NELKEN (2010), pp.136; DÍEZ-RIPOLLÉS (2011), pp. 1-36; DÍEZ-RIPOLLÉS (2013), pp. 62-78, entre otros.
} 


\section{Polít. Crim. Vol. 15, N 30 (Diciembre 2020), Art. 6, pp. 670-693 [http://politcrim.com/wp-content/uploads/2020/12/Vol15N30A6.pdf]}

alejamiento de los diversos sistemas de control penal de un estricto enfoque socialmente excluyente.

A este modelo analítico subyacen dos presupuestos: La producción o reforzamiento de la exclusión social de los citados colectivos por los órganos de persecución penal genera mayor delincuencia a mediano y largo plazo. La obtención de un significativo nivel de inclusión social de esos colectivos reduce la delincuencia a mediano y largo plazo. Ambos presupuestos están pendientes de demostración empírica, y nuestra investigación no los plantea como hipótesis a verificar. Lo que pretendemos es construir, y posteriormente aplicar, un instrumento de comparación internacional que permita clasificar sistemas de control penal nacionales a tenor de sus efectos socialmente excluyentes de acuerdo con ese modelo teórico. Ello habrá de sentar las bases para probar en el futuro la corrección o no de los presupuestos antedichos.

El instrumento que ha de hacer operativo el modelo teórico debe estar constituido por un conjunto de indicadores que nos permitan medir el grado en que diferentes sistemas penales nacionales producen efectos socialmente excluyentes sobre los colectivos referidos. Tales indicadores deberán poseer las siguientes características descritas a continuación.

Irán referidos a reglas y prácticas punitivas reales, es decir, que son objeto de efectiva aplicación en países del mundo occidental desarrollado. Por regla punitiva se entiende una norma jurídica, usualmente aunque no siempre inserta en el ordenamiento jurídico-penal, que prevé determinadas consecuencias frente a ciertos comportamientos o situaciones relacionados con el control penal. Por práctica punitiva se entiende la forma en que efectivamente reaccionan ante comportamientos o situaciones relacionados con el control penal diversas agencias sociales, de acuerdo o no con lo previsto legalmente. Se asume que se pueden producir solapamientos entre los contenidos de ciertas reglas y prácticas, lo que se tendrá en cuenta al configurar el instrumento.

El criterio primario de selección de las reglas y prácticas punitivas es su robusta capacidad para medir efectos de exclusión social significativos sobre los colectivos estudiados. En ningún caso se pretenden identificar reglas y prácticas punitivas que midan la producción de efectos relevantes de inclusión social sobre los citados colectivos.

El conjunto de indicadores escogidos habrá de reflejar de manera comprensiva la realidad del modelo político-criminal de los respectivos países en lo que concierne a sus efectos socialmente excluyentes sobre los colectivos objetos de estudio. A estos efectos, el modelo teórico de Díez-Ripollés identifica nueve grandes áreas de intervención penal especialmente aptas para mostrar efectos excluyentes relevantes, y que en su conjunto dan una imagen comprensiva del correspondiente sistema penal. Las nueve áreas o cestas son: control de espacios públicos, garantías penales, sistemas de penas y de su determinación, penas máximas, régimen penitenciario, internamientos de seguridad, estatus legal y social de condenados y excondenados, registros policiales y penales y derecho penal juvenil. A su vez esas nueve cestas de reglas y prácticas punitivas pueden descomponerse en un total de 
veinticinco subgrupos o manojos. ${ }^{4}$ En consecuencia, todos los indicadores a integrar en el instrumento deberán poder integrarse en una de esas cestas y manojos.

Los indicadores finalmente escogidos tendrán capacidad discriminatoria, esto es, mostrarán variaciones relevantes en los diversos sistemas penales del mundo occidental desarrollado.

El instrumento se construirá sobre el acuerdo existente entre destacados expertos pertenecientes a diferentes países del mundo occidental desarrollado de que las reglas y prácticas punitivas seleccionadas producen de manera significativa exclusión social sobre alguno de los siguientes colectivos: sospechosos/procesados, condenados y excondenados. En ningún momento se pretende verificar empíricamente a través de trabajos de campo que tales reglas o prácticas punitivas generan efectivamente exclusión social. Esa verificación se sustituye por su validación a través de un amplio consenso entre expertos.

Una vez creado el instrumento RIMES se procederá a su aplicación en los países occidentales desarrollados. Cuando ya se disponga de resultados de un número significativo de países se procederá a hacer comparaciones sobre los diversos modelos políticocriminales nacionales vigentes a partir de una dimensión muy significativa, la de la exclusión social que generan en colectivos especialmente afectados por la intervención penal. Entre otras actuaciones, se procederá a insertar a los países en una escala que mida su puntuación, global o parcial, obtenida en el conjunto de indicadores del instrumento o en los indicadores agrupados por cestas. La información obtenida será de gran utilidad para el conocimiento e interpretación de la política criminal contemporánea, así como para la toma de decisiones político-criminales fundamentadas.

\section{Objetivos}

El objetivo general del proyecto es hacer operativo el modelo teórico aludido mediante el desarrollo y aplicación de un instrumento capaz de medir, en términos comparativos, la exclusión social generada sobre sospechosos, procesados, condenados y excondenados por los actuales sistemas de control penal de los países occidentales desarrollados.

Los objetivos específicos del proyecto son los siguientes:

1) Identificar un conjunto de reglas y prácticas punitivas generadoras de exclusión social, que resulten además aptas para constituir el instrumento de medición comparativa.

\footnotetext{
4 Que son los siguientes: Control de espacios públicos (urbanizaciones cerradas, videovigilancia, prohibiciones urbanas de acceso), garantías penales (socavamiento de garantías penales, obstaculización o restricción de recursos judiciales), sistemas de penas y de su determinación (discreción judicial, leyes agravadas de reincidencia, uso extensivo de prisión, sanciones alternativas a prisión, controles electrónicos), penas máximas (pena de muerte, cadena perpetua, penas largas de prisión), régimen penitenciario (condiciones de vida en prisión, respeto de derechos de reclusos, libertad condicional), internamientos de seguridad (prisión preventiva, internamientos tras liquidación condena), estatus legal y social de condenados y excondenados (privación de derechos de participación política, privación de otros derechos civiles, recursos sociales accesibles), registros policiales y penales (expansión y accesibilidad de registros, paradero de excondenados), derecho penal juvenil (límites de edad, tratamiento diferenciado de adultos).
} 


\section{Polít. Crim. Vol. 15, No 30 (Diciembre 2020), Art. 6, pp. 670-693 \\ [http://politcrim.com/wp-content/uploads/2020/12/Vol15N30A6.pdf]}

2) Validar las reglas y prácticas resultantes de la selección anterior mediante un método de validez de contenido por juicio de expertos, que combina rasgos cualitativos y cuantitativos y que implica la colaboración de numerosos expertos internacionales. Ese proceso de validación presumiblemente reducirá de forma significativa el número de reglas y prácticas punitivas sobrevivientes. De entre ellas se escogerán, atendiendo a criterios operativos adicionales, las que se configurarán como indicadores del definitivo instrumento de medición comparativa RIMES.

\section{Elaboración del instrumento de medición comparativa RIMES}

Para la creación y validación del instrumento RIMES se ha procedido a una estrategia metodológica cualitativa a través del método Delphi ${ }^{5}$ y de la técnica de validación de contenidos por juicio de expertos. ${ }^{6}$ Se escoge esta metodología porque es la más adecuada para alcanzar un consenso sobre el contenido y la validez del instrumento a través de un proceso sistemático, formal y profundo. Se recomienda esta aproximación cuando no se dispone de información suficiente para tomar decisiones. ${ }^{7}$

En la selección previa de reglas y prácticas punitivas el grupo de expertos ha estado compuesto por la mayoría de los integrantes del área de Derecho Penal y del Instituto de Criminología de la Universidad de Málaga, a los que se les aplicó el método Delphi para comprobar el grado de pericia en las temáticas a abordar. Los resultados fueron satisfactorios, pues, aunque incluye algunas personas en proceso de formación doctoral, la mayor parte del grupo abarca un número suficiente de personas con grado de doctor que se consideran expertas en más de una cesta. Ello permite cubrir toda la extensión de conocimientos que se quiere abarcar.

En la validación del RIMES, hasta conseguir un instrumento adecuado, se ha procedido a un juicio de expertos, en el que se ha contado con 97 expertos internacionales de 18 países diferentes.

Los procedimientos llevados a cabo para seleccionar ítems, elegir a los expertos y validar el instrumento se exponen a continuación.

\subsection{Primera fase. Formulación inicial del instrumento}

Entre marzo y diciembre de 2013 un equipo de investigación constituido por 19 expertos en política criminal, Derecho Penal o criminología afiliados al Departamento de Derecho Penal y al Instituto de criminología de la Universidad de Málaga desarrolló los trabajos para identificar un conjunto de reglas y prácticas punitivas que consideran especialmente

\footnotetext{
${ }^{5}$ CRUZ (2009), passim.

${ }^{6}$ ESCOBAR-PÉREZ y CUERVO-MARTÍNEZ (2008), pp. 27-36; CABERO y BARROSO (2013), pp. 2538.

${ }^{7}$ REGUANT-ÁLVAREZ y TORRADO-FONSECA (2016), pp. 87-102.
} 
generadoras de exclusión social en los colectivos señalados de acuerdo con el modelo teórico de Díez Ripollés.

Los integrantes del equipo se distribuyeron entre las nueve cestas temáticas atendiendo a sus ámbitos de especialización y a lograr un equilibrio entre personas con mayor o menor experiencia. De cada cesta se ocupan entre dos o tres personas, habiendo casos en que un experto participa en más de una cesta. Un equipo coordinador se ocupa de atender posibles dudas metodológicas y gestionar el proceso.

Entre las instrucciones impartidas están las referidas a las características que han de tener los ítems a identificar, ya señaladas en el marco teórico, a las que se añaden las siguientes:

1) Las reglas y prácticas punitivas se formularán siempre en términos positivos, es decir, expresando su potencialidad socialmente excluyente, y se admitirá solo una respuesta dicotómica Si o No.

2) Se procurará lograr una proporción aceptable entre reglas y prácticas.

3) Se intentará que esas reglas y prácticas se distribuyan de una manera limitadamente uniforme entre las diversas cestas del modelo teórico que inspira el proyecto.

4) A la hora de seleccionar las reglas y prácticas punitivas se habrán de tener en cuenta datos de política criminal comparada.

5) Aunque en los documentos iniciales de trabajo se podrá utilizar el español o el inglés, se ha de tener en cuenta que el instrumento a validar en la segunda fase deberá estar formulado en inglés, redacción que constituirá la única versión válida.

Cada uno de los nueve grupos solo presentará al conjunto del equipo de investigación aquellas reglas y prácticas en las que se haya alcanzado consenso entre los integrantes del grupo.

Durante ese período se produjeron numerosas reuniones de los miembros de cada cesta y numerosas consultas de los integrantes de cada cesta al equipo coordinador. Paulatinamente, a medida que los trabajos de cada cesta se van concluyendo, se mantienen 18 reuniones del equipo de investigación en pleno, en las que se revisan críticamente las reglas y prácticas propuestas para cada cesta, y se da el visto bueno por asentimiento de todo el equipo a las finalmente seleccionadas. El resultado de esa labor es la identificación de 278 reglas y prácticas punitivas, distribuidas por las nueve cestas.

A partir de esa primera selección los miembros del equipo coordinador proceden a un análisis detenido de las citadas reglas y prácticas a los efectos de verificar solapamientos de contenido, totales o parciales, entre reglas y prácticas de una misma cesta o localizadas en diversas cestas. Ello resulta en una primera reducción del número de ítems (reglas y prácticas) del instrumento proyectado hasta un número de 250. 


\section{Polít. Crim. Vol. 15, No 30 (Diciembre 2020), Art. 6, pp. 670-693 [http://politcrim.com/wp-content/uploads/2020/12/Vol15N30A6.pdf]}

Posteriormente los cuatro miembros del equipo coordinador, en febrero de 2014, acuerdan confrontar cada uno de los ítems con tres criterios coherentes con los presupuestos teóricos y metodológicos que han de fundar el instrumento. Los tres criterios son los siguientes:1) exhaustividad, que expresa la especial relevancia de la información que suministra 2) extensión, que asegura que la información no se ocupa de asuntos en exceso particulares o específicos y 3) facilidad, en virtud del cual se prevé que la información va a ser relativamente sencilla de obtener.

Cada ítem se puntúa 1 o 0 en cada uno de los criterios en función de si se estima que lo cumple o no. Acumuladas las puntuaciones de todos los miembros del equipo, se repasa cada ítem y se decide excluir todos aquellos que no hayan superado los 4 puntos (uno por cada uno de los miembros del equipo coordinador) en el conjunto de los criterios. El resultado es que superan esas exigencias 221 ítems.

A continuación, entre marzo y abril de 2014, se decidió someter al conjunto de ítems que superaron esa primera revisión a tenor de los tres criterios mencionados, a una nueva por parte del mismo equipo coordinador. En este caso, además de proponer una más estricta aplicación de los criterios ya utilizados, se añadieron dos nuevos: 1) capacidad de discriminación, de modo que el ítem permita establecer diferencias entre los países objeto de estudio y 2) claridad, esto es, facilidad de comprensión del ítem.

Las puntuaciones en cada uno de los criterios, así como su acumulación se realizaron como en la revisión anterior. Obtenidos los resultados, se adoptaron por el conjunto del equipo coordinador las siguientes decisiones: 1) Mantener todos los ítems con una puntuación acumulada en cada uno los cinco criterios de 3 o 4. 2) Excluir los ítems que obtuvieran una puntuación acumulada de 0 o 1 en exhaustividad o en discriminación. 3) Evaluar los ítems cuyas puntuaciones acumuladas no se encontraron en ninguna de las situaciones anteriores. 4) Aplicar el criterio de la extensión tomando como referencia las cestas en las que se integran los diferentes ítems, es decir, se intentaba evitar que una cesta tuviera demasiados ítems referidos a un mismo asunto. 5) Asegurar que hubiera una aceptable proporción entre reglas y prácticas dentro de cada cesta.

Aplicados esos criterios se obtiene como resultado un total de 126 ítems (véanse en tabla ${ }^{\circ}$ 7 distribuidos de una manera homogénea entre las nueve cestas ${ }^{8}$ ), de los cuales 79 son reglas y 47 prácticas punitivas.

Ha de señalarse que durante las dos revisiones realizadas por el equipo coordinador se producen ocasionalmente mejoras en la formulación de los ítems que permanecen y se siguen descartando ítems que dan lugar a solapamientos parciales.

\subsection{Segunda fase. Validación del instrumento}

En esta segunda fase se pretende validar el instrumento hasta entonces obtenido mediante el método de validez de contenido por juicio de expertos. Ya que se pretende crear un

\footnotetext{
${ }^{8}$ Contienen cada una entre 12 y 15 ítems, salvo una cesta que tiene 19.
} 
instrumento de medición comparativa aplicable a los países occidentales industrializados, la validación del mismo exigía la colaboración de numerosos expertos del mundo occidental desarrollado, por lo que una buena parte del esfuerzo se ha destinado a la elección de este selecto grupo.

3.2.1. Versión definitiva de instrumento a validar, selección de expertos y creación de los cuestionarios de validación

Entre fines de 2014 y comienzos de 2015 se pasan a realizar diversas tareas de gran importancia, preparatorias del proceso de validación.

1) Una vez fijados los ítems a someter a validación por expertos, se formula la versión inglesa de esos ítems, parcialmente ya realizada en fases precedentes, pero que precisa de una versión definitiva. Tras realizar una primera versión inglesa de los 126 ítems, se encomienda su revisión a una jurista/criminóloga de habla inglesa. Tras diversas comunicaciones con ella, con las que se pretende, sobre todo, asegurar que no se hacen formulaciones negativas de los ítems y que se han seleccionado los términos técnicamente más adecuados para expresar los diversos ítems, la versión inglesa definitiva está lista a final de año.

2) Se emprenden las tareas de seleccionar los expertos que han de validar el instrumento, entrar en contacto con ellos y conseguir su aceptación.

Previamente hubo que determinar los criterios de selección de los expertos. Tras un intenso debate entre los miembros del equipo coordinador, y con el asesoramiento de las metodólogas, los criterios definitivamente fijados son los siguientes:

1) Los expertos deben realizar su desempeño profesional en países del mundo occidental desarrollado, y entre todos deberán cubrir las cinco siguientes áreas geográficas: Europa nórdica, Europa central, Europa oriental, Europa meridional, países anglosajones europeos y extraeuropeos.

2) Los expertos deben desenvolverse en el ámbito de la política criminal, el Derecho Penal o la criminología, y poseer una acreditada y significativa experiencia en actividades académicas o profesionales referidas a la prevención o persecución de delitos. Esa experiencia deberá extenderse por un período superior a los 10 años. Los expertos académicos deberán ser doctores. ${ }^{9}$

3) Deberá asegurarse un moderado equilibrio entre académicos y profesionales, hombres y mujeres, y orientación política.

a) La procedencia de esos expertos se distribuirá entre países de las áreas geográficas indicadas de modo que ningún país adquiera una sobrerrepresentación sobre otros, sin perjuicio de que los países con una población más pequeña aporten menos expertos.

\footnotetext{
${ }^{9}$ Inicialmente se estableció esta exigencia también para los profesionales, pero a lo largo de la selección de
} los expertos se abandonó esta exigencia para los no académicos. 


\section{Polít. Crim. Vol. 15, № 30 (Diciembre 2020), Art. 6, pp. 670-693 [http://politcrim.com/wp-content/uploads/2020/12/Vol15N30A6.pdf]}

b) Se intentará obtener en torno a 100 expertos pertenecientes a 20 países distintos.

A partir de esos criterios, se procedió a una primera identificación de académicos y profesionales que constituyeran una referencia internacional desde sus respectivos países en los asuntos más arriba indicados. Se procuró encontrar dos expertos por país, aunque se asumía que en países pequeños podía ser suficiente con uno. Dada la relevancia de las personas buscadas, en la mayoría de las ocasiones ya eran conocidas de antemano entre los miembros del equipo de investigación. En unos pocos casos se consultaron fuentes bibliográficas, aportaciones a congresos, membresía en ciertas organizaciones y otros elementos de juicio. Se trataba en todos los casos de personas con una larga carrera académica o profesional a sus espaldas, por lo que su experiencia superaba con holgura los 20 años. Se procuró que la selección realizada fuera equilibrada a tenor de las exigencias antes señaladas.

A continuación, nos pusimos en contacto con esas personas, a las que se les informaba del proyecto en curso, se les preguntaba si deseaban participar y se les indicaba las tres contribuciones que se les iba a pedir en caso de que aceptaran. La primera era rellenar un breve cuestionario con algunas informaciones personales. La segunda consistía en dar tres nombres de colegas de su propio país que a su juicio poseyeran la experiencia académica o profesional exigidas para participar en el proyecto. La tercera era participar en la segunda fase de validación del cuestionario, la cual iría referida a los ítems que hubieran superado la primera fase de validación. No todos los expertos inicialmente conectados aceptaron participar, aunque sí un número significativo de ellos, por lo que se siguieron estableciendo contactos con otros expertos de sus respectivos países que cumplieran las condiciones antedichas. Finalmente, tras conectar con 58 expertos de 20 países, aceptaron participar en el primer pase 28 de 18 países $^{10}$ (véase tabla 1). Con todos ellos se volvió a entrar en contacto para que cumplimentaran las dos primeras contribuciones solicitadas.

A medida que se iba avanzando en la obtención de este colectivo de expertos y se les pedían las dos primeras contribuciones, se recogían igualmente, mediante la técnica de bola de nieve, los nombres de los tres expertos de su propio país que proponían. Estos nuevos expertos eran examinados por los miembros del equipo coordinador para verificar que aisladamente o en conjunto satisfacían los criterios fijados para todos los expertos. La mayoría de los primeros expertos suministraron los tres nombres solicitados, pero en ocasiones ofrecieron menos o ninguno. Además, había casos en los que los expertos suministrados no cumplían el perfil buscado o, aun cumpliéndolo, debían ceder el paso a otros con perfiles que permitían completar el colectivo ajustado a nuestros propósitos. Finalmente, un número determinado de expertos de este segundo grupo declinó participar en el proyecto una vez remitida la carta en la que se les informaba de la naturaleza del proyecto y de la contribución que se les solicitaba en la primera fase de validación del proyecto, y unos pocos, pese a aceptar participar, no lo terminaron haciendo. En todos estos casos, bien nos poníamos de nuevo en contacto con los primeros expertos pidiéndoles nuevos nombres, bien procedíamos a su búsqueda con medios similares a los empleados con los primeros expertos seleccionados. Se procuró en la medida de lo posible que se

\footnotetext{
${ }^{10}$ Dos de ellos finalmente no pudieron participar en la validación del cuestionario por razones mencionadas infra. No se consiguió obtener participación de expertos procedentes de Austria y Suecia.
} 


\section{DÍEZ RIPOLLÉS, José Luis; GARCÍA, Elisa: "RIMES: Un instrumento de comparación de políticas criminales nacionales desde la exclusión social"}

mantuviera la ratio de tres nuevos expertos del mismo país por cada uno de los expertos inicialmente seleccionados, lo que no siempre se consiguió. En último término, aunque partimos de un total de 84 expertos que inicialmente habían aceptado, pudimos efectivamente disponer de un total de 71 nuevos expertos de los mismos 18 países (véase tabla 1) a los que pertenecían los expertos primeramente seleccionados.

En suma, la selección de expertos se materializó en la efectiva participación en la validación del cuestionario de 97 expertos de 18 países, como se muestra en la tabla 1. Dada la metodología seguida en la validación del instrumento conviene mencionarlos, a los efectos de mostrar su prestigio, relevancia y variedad dentro de la política criminal, el derecho penal y la criminología.

Tabla 1. Expertos seleccionados en los dos pases según países

\begin{tabular}{|c|c|c|}
\hline País & Expertos Pase 2 & Expertos Pase 1 \\
\hline Alemania & $\begin{array}{l}\text { Frieder Dünkel } \\
\text { Rita Haverkamp }\end{array}$ & $\begin{array}{l}\text { Ineke Pruin } \\
\text { Christine Morgenstern } \\
\text { Harald Arnold } \\
\text { Tillmann Bartsch }\end{array}$ \\
\hline Bélgica & $\begin{array}{l}\text { Stephan Parmentier } \\
\text { Sonja Snacken }\end{array}$ & $\begin{array}{l}\text { Tom Daems } \\
\text { Jenneke Christiaens } \\
\text { Els Dumortier } \\
\text { Sofie De Kimpe }\end{array}$ \\
\hline Canadá & Anthony Doob & $\begin{array}{l}\text { Cheryl Webster } \\
\text { Howard Bebbington } \\
\text { Jane B. Sprott }\end{array}$ \\
\hline Dinamarca & Anette Storgaard & $\begin{array}{l}\text { Signe Herving } \\
\text { Ditte Nyggard } \\
\text { Louise Hansen }\end{array}$ \\
\hline EE.UU & $\begin{array}{l}\text { Emilio Viano } \\
\text { James Jacobs }\end{array}$ & $\begin{array}{l}\text { Clairissa Breen } \\
\text { Alison Mikkor } \\
\text { Katharine Tinto } \\
\text { Elisabeth Nevins } \\
\text { Lila Kazemian }\end{array}$ \\
\hline \multirow[t]{2}{*}{ España } & Elena Larrauri & $\begin{array}{l}\text { Ester Blay Gil } \\
\text { Lorena Antón García } \\
\text { Josep Cid Moliné } \\
\text { Juan José Medina Ariza }\end{array}$ \\
\hline & José Luis de la Cuesta & $\begin{array}{l}\text { Ana Isabel Perez Machio } \\
\text { Isabel Germán Mancebo } \\
\text { Xabier Arana Berastegi }\end{array}$ \\
\hline Finlandia & Tapio Lappi-Seppälä & $\begin{array}{l}\text { Mikko Aaltonen } \\
\text { Janne Kivivuori } \\
\text { Henrik Linderborg }\end{array}$ \\
\hline
\end{tabular}


Polít. Crim. Vol. 15, № 30 (Diciembre 2020), Art. 6, pp. 670-693

[http://politcrim.com/wp-content/uploads/2020/12/Vol15N30A6.pdf]

\begin{tabular}{|c|c|c|}
\hline Francia & Laurence Dumoulin & $\begin{array}{l}\text { Jacques DeMaillard } \\
\text { Sebastian Roché }\end{array}$ \\
\hline Holanda & $\begin{array}{l}\text { John Vervaele } \\
\text { René Van Swaaningen }\end{array}$ & $\begin{array}{l}\text { Michiel Lutchtman } \\
\text { Jolande uit Biejerse } \\
\text { Marijke Malsch } \\
\text { John Blad }\end{array}$ \\
\hline Hungría & Miklós Levay & $\begin{array}{l}\text { Zoltan Fleck } \\
\text { Eszter Sárik } \\
\text { Szilveszter Póczik }\end{array}$ \\
\hline Inglaterra & $\begin{array}{l}\text { Nicole Padfield } \\
\text { Gillian Mclvor }\end{array}$ & $\begin{array}{l}\text { Jane Dominey } \\
\text { Wendy Fitzgibbon } \\
\text { Joel Miller } \\
\text { Niall Hamilton-Smith } \\
\text { Paul Quinton }\end{array}$ \\
\hline Irlanda & Michael Reilly & $\begin{array}{l}\text { Eimear Spain } \\
\text { Mary Rogan } \\
\text { Claire Hamilton }\end{array}$ \\
\hline Italia & $\begin{array}{l}\text { Lorenzo Picotti } \\
\text { Fabio Quassoli }\end{array}$ & $\begin{array}{l}\text { Roberto Flor } \\
\text { Ivan Salvadori } \\
\text { Roberto Cornelli } \\
\text { Alvise Sbraccia } \\
\text { Andrea Molteni }\end{array}$ \\
\hline Nueva Zelanda & Warren Young & $\begin{array}{l}\text { John Pratt } \\
\text { Yvette Tinsley }\end{array}$ \\
\hline Polonia & $\begin{array}{l}\text { Beata Gruszczynska } \\
\text { Krzysztof Krajewsky }\end{array}$ & $\begin{array}{l}\text { Pawel Ostaszewski } \\
\text { Bárbara Błońska } \\
\text { Grzegorz Bogdan } \\
\text { Krzysztof Wilamowski } \\
\text { Witold Klaus }\end{array}$ \\
\hline Portugal & $\begin{array}{l}\text { Candido Da Agra } \\
\text { Augusto Silva Dias }\end{array}$ & $\begin{array}{l}\text { Diogo Da Costa } \\
\text { Jorge Quintas } \\
\text { José Leal } \\
\text { Inês Ferreira Leite } \\
\text { Ana Sofia Xavier Marques }\end{array}$ \\
\hline Rumanía & Florin Streteanu & $\begin{array}{l}\text { Daniel Nitu } \\
\text { Sergiu Bogdan } \\
\text { Raluca Morosanu }\end{array}$ \\
\hline Suiza & $\begin{array}{l}\text { Marcelo Aebi } \\
\text { Nicolás Queloz }\end{array}$ & $\begin{array}{l}\text { Giang Ly Isenring } \\
\text { Manon Jendly } \\
\text { Anne-Claude Scheidegger } \\
\text { Frédéric Gisler } \\
\text { Raphael Brossard }\end{array}$ \\
\hline
\end{tabular}


3) El diseño del cuestionario mediante el que se iba a proceder a la validación de los 126 ítems inicialmente seleccionados, y su proceso de aplicación, se aprobó por el equipo coordinador en febrero de 2015 en los términos siguientes:

1. El cuestionario de ítems se validará tras dos pases entre el colectivo de expertos seleccionados. En el primer pase participarán 84 expertos, todos aquellos que fueron seleccionados en la segunda fase de selección de expertos más arriba mencionada. En el segundo pase participarán los 28 expertos que fueron inicialmente seleccionados.

2. En el primer pase se someterán a validación por los expertos el total de 126 ítems que resultaron tras la identificación, selección y revisión de las reglas y prácticas punitivas generadoras de exclusión social por parte del equipo de investigación. Cada uno de los expertos se ocupará de un tercio de los ítems incluidos en el cuestionario inicial, lo que da lugar a que el cuestionario se divida en tres bloques de 42 ítems. A los expertos les corresponderá hacer el cuestionario $1-$ que contempla los ítems 1 a 42 del cuestionario completo-, 2 -que incluye los ítems 43 a 84 del cuestionario completo-, o 3 -que abarca los ítems 85 a 126 del cuestionario completo - de modo aleatorio, asignándoles sucesivamente uno de los tres según el momento en que confirmen su participación en el proceso de validación.

3. Los cuestionarios parciales que se cumplimentarán en el primer pase constarán de los siguientes elementos: Una primera parte en la que se expondrán brevemente los conceptos básicos de lo que se quiere medir, con ejemplos, y las instrucciones para contestar adecuadamente a las preguntas que se hacen en el cuestionario de ítems que se adjunta. Estas preguntas serán dos: La primera se referirá a la claridad del correspondiente ítem, esto es, a si se comprende bien su contenido semántico. La segunda aludirá a la idoneidad de la regla o práctica punitiva mencionada en el ítem para generar exclusión social en los colectivos que son objeto de estudio. Cada una de las dos preguntas formuladas para cada ítem deberá puntuarse en una escala Likert de 1 a 5 , en el que 1 es el valor inferior en claridad o idoneidad y 5 el superior en esas dimensiones. La segunda parte contendrá la lista de ítems, la cual permitirá contestar en una misma línea a las dos preguntas de cada uno de ellos. La tercera parte contendrá un formulario a rellenar por el experto en el que se incluirán una serie de preguntas personales y profesionales similares a las ya formuladas al primer grupo de expertos tras su aceptación a participar. Los cuestionarios deberán ser cumplimentados en línea mediante el programa Qualtrics, que es una plataforma de investigación para la toma de decisiones complicadas, de fácil diseño y ejecución, a cuyos efectos se hará llegar el enlace correspondiente mediante un correo electrónico a cada uno de los expertos.

4. En el segundo pase se someterán a validación del segundo grupo de expertos los ítems que hayan superado los criterios de validación del primer pase. En este caso el cuestionario completo resultante, si las estimaciones respecto a su tamaño se confirman, se dividirá en dos, asignando una u otra mitad del cuestionario a los expertos con criterios a determinar más adelante. Esto es, cada parte del cuestionario será realizada por 14 expertos. Si los ítems que superan el primer pase fueran inferiores a 40, se aplicaría el cuestionario completo resultante a cada uno de los integrantes de este grupo de expertos. 


\section{Polít. Crim. Vol. 15, No 30 (Diciembre 2020), Art. 6, pp. 670-693 [http://politcrim.com/wp-content/uploads/2020/12/Vol15N30A6.pdf]}

5. Los cuestionarios a cumplimentar en el segundo pase constarán de los siguientes elementos: una primera parte semejante a la de los cuestionarios del primer pase, salvo que se incluye una información adicional sobre las nueve cestas en las que se distribuyen el total de ítems del instrumento a validar. Ello se debe a que las preguntas a responder ya no son dos sino tres. A las dos ya contenidas en los cuestionarios del primer pase se añade una tercera, en la que se inquiere sobre la adecuación de incluir el ítem correspondiente en una determinada cesta. Las tres preguntas deberán puntuarse en una escala Likert de 1 a 5 , del mismo modo que en el pase anterior. La segunda parte del cuestionario contendrá la lista de ítems, disponiendo cada uno de ellos de tres líneas para contestar a las tres preguntas. No se incluirá una tercera parte similar a la del cuestionario del primer pase, pues esa información ya se habrá obtenido en un momento previo, como ya se ha dicho.

Los cuestionarios se harán llegar a los expertos y deberán ser cumplimentados igual que en el pase precedente.

\subsubsection{Validación del cuestionario por expertos. Primer pase}

Los plazos dados inicialmente para cumplimentar los cuestionarios 1,2 y 3 del primer pase oscilaron entre uno y dos meses. No obstante, la práctica mostró que esos plazos eran muy optimistas, pues la cumplimentación y recogida de estos cuestionarios se extendió a lo largo de un período de 15 meses, hasta mayo de 2016. A la tardanza en contestar de un grupo significativo de estos expertos, lo que obligó a enviar, en el peor de los casos, hasta tres recordatorios, se unió que otros expertos, pese a haber aceptado, finalmente no contestaron y tuvimos que buscar nuevos expertos de los países correspondientes, al margen de otras incidencias. Finalmente, el cuestionario 1 fue cumplimentado por 26 expertos de 18 países, el cuestionario 2 por 23 expertos de 17 países y el cuestionario 3 por 22 expertos de 16 países. Es decir, se consiguió una tasa de respuesta del $84,52 \%$ del total de cuestionarios enviados; quedaron sin cumplimentar dos cuestionarios 1 , cinco cuestionarios 2 y seis cuestionarios 3 .

Una vez en nuestro poder las respuestas a los cuestionarios, las analizamos a través de la prueba estadística de fiabilidad inter-jueces consistente en el coeficiente de validación $\mathrm{V}$ de AIKEN. ${ }^{11}$ A partir de este coeficiente se consideraron en principio no validados todos los ítems que tuvieran un coeficiente inferior a 0,70. Eso daba lugar a que, del total de 126 ítems sometidos a validación, en idoneidad resultaran validados 81 ítems, de los cuales 62 también se validaban en claridad (véanse Tablas 2 y 3 ).

\footnotetext{
${ }^{11}$ AIKEN (1980), pp. 955-959.
} 
Tabla 2. Resultados en idoneidad según coeficiente V de Aiken

\begin{tabular}{|l|c|}
\hline \multicolumn{1}{|c|}{ Intervalos del coeficiente V de Aiken } & n \\
\hline 0.80 y más & 42 \\
\hline $0.75-0.79$ & 19 \\
\hline $0.70-0.74$ & 20 \\
\hline No válidos & 45 \\
\hline TOTAL & 81 \\
\hline
\end{tabular}

Tabla 3. Resultados en claridad según coeficiente V de Aiken de los 81 ítems idóneos

\begin{tabular}{|c|c|c|}
\hline Problemas de claridad & Intervalos del coeficiente V de Aiken & n \\
\hline Ningún problema & 0,70 y más & 62 \\
\hline Algún problema & $0,60-0,69$ & 13 \\
\hline Con problemas & $0,50-0,59$ & 6 \\
\hline
\end{tabular}

Teniendo en cuenta estos resultados, el equipo de investigación puso en práctica las siguientes decisiones, inspiradas en mejorar la operatividad del instrumento en elaboración:

1) Utilizar como punto de referencia para configurar el cuestionario del segundo pase los ítems que hubieran obtenido unos coeficientes de Aiken en idoneidad y en claridad no inferiores a 0,70 .

2) Permitir la inclusión en el cuestionario del segundo pase de algunos ítems que, habiendo obtenido en claridad un coeficiente entre 0,55 y 0,69 , tuvieran en todo caso en idoneidad un coeficiente de Aiken no inferior a 0,70 y fueran necesarios para satisfacer los otros criterios que siguen.

En el caso de que finalmente sean incorporados al cuestionario del segundo pase de validación, se hará previamente un esfuerzo en mejorar su redacción para que ganen en claridad.

3) Reducir, de cara al segundo pase, el número de ítems del cuestionario. A tales efectos se revisarán todos los ítems con las cualidades más arriba indicadas de acuerdo con los siguientes cuatro criterios:

a) Redundancia del ítem, es decir, grado en que se ocupa, aunque no haya solapamiento de contenido, de un asunto similar a otro de la misma cesta.

b) Búsqueda de cierta proporción entre ítems que constituyan regla o práctica punitiva dentro de cada cesta y en el cuestionario en general.

c) Mantenimiento de un número de ítems por cesta no muy disparejo.

d) Mayor o menor puntuación en claridad. 
El resultado de toda esa labor es un cuestionario de 65 ítems para el segundo pase, que se distribuye entre nueve cestas, teniendo cada una de ellas entre 5 y 11 ítems, y suponiendo un total de 44 reglas y 21 prácticas.

\subsubsection{Validación del cuestionario por expertos. Segundo pase.}

Una vez conocida la estructura y extensión del cuestionario que va a ser sometido a validación por los expertos del segundo pase, el equipo investigador, entre septiembre y octubre de 2016, adopta las siguientes decisiones metodológicas adicionales:

1) El cuestionario se dividirá en dos: El cuestionario 1 constará de 33 ítems, y el cuestionario 2 tendrá 32 ítems. Los ítems se distribuyen de modo aleatorio entre los dos cuestionarios parciales a cumplimentar.

2) Los expertos de este segundo pase se dividen en dos subgrupos, de modo que uno responderá al cuestionario 1 y el otro al cuestionario 2. La división de los expertos en dos subgrupos no se hace de un modo aleatorio, pues se pretende que exista una heterogeneidad grupal equivalente en base a los rasgos personales que ya se obtuvieron de cada uno de estos expertos.

Los dos cuestionarios se envían a fines de octubre de 2016. A finales de ese año se concluye la recogida de los cuestionarios enviados, con una tasa de respuesta muy alta, del $92,85 \%$, pues se cumplimentan 26 de los 28 cuestionarios remitidos, 13 del cuestionario 1 y 13 del cuestionario $2 .{ }^{12}$

Aplicado el coeficiente de validación V de Aiken a los ítems de los dos cuestionarios se obtienen en claridad coeficientes superiores a 0,60 en todos los ítems, en idoneidad se alcanza o supera un coeficiente de 0,70 en 55 de los 65 ítems, y en adecuación 63 de los 65 ítems igualan o superan el coeficiente de 0,70 (véase Tabla 4).

Tabla 4. Resultados del coeficiente V de Aiken

\begin{tabular}{|c|c|c|c|}
\hline $\begin{array}{c}\text { Intervalos del coeficiente V de } \\
\text { Aiken }\end{array}$ & Claridad & Idoneidad & $\begin{array}{c}\text { Adecuación por } \\
\text { cestas }\end{array}$ \\
\hline 0.80 y más & 45 & 35 & 56 \\
\hline $0.75-0.79$ & 12 & 13 & 5 \\
\hline $0.70-0.74$ & 1 & 7 & 2 \\
\hline 0.69 y menos & 7 & 10 & 1 \\
\hline No valido & 0 & 0 & 1 \\
\hline TOTAL & 65 & 65 & 64 \\
\hline
\end{tabular}

\footnotetext{
${ }^{12}$ Uno de los cuestionarios pendientes no se recibe por fallecimiento del experto, y el otro por falta de tiempo ligada a nombramiento para un cargo relevante de gestión académica.
} 
Hecho el descarte de todos los ítems con valores inferiores a 0,70 en el coeficiente $\mathrm{V}$ de Aiken en idoneidad y adecuación obtenemos como resultado un cuestionario constituido por 55 ítems. De estos, solo tres ítems obtienen en claridad un coeficiente de Aiken entre 0,63 y 0,69 , teniendo el resto un coeficiente de Aiken igual o superior a 0,70 .

\subsubsection{Configuración final del instrumento RIMES}

A comienzos de 2017 el equipo de investigación constata con satisfacción que tras los dos pases de validación se ha logrado que los expertos validaran un número significativo de ítems, 55, de los 126 que iniciaron el proceso de validación inter-jueces.

Con todo, el número de ítems validados resulta excesivo de cara a una factible aplicación del instrumento, por lo que se decide realizar una nueva revisión de los 55 ítems por todo el equipo, en la que se atenderá a los siguientes criterios:

1) Procurar que el instrumento final esté compuesto por un número de ítems en torno a 35 , sin superar en ningún caso el número de 40.

2) Preservar en la medida de lo posible aquellos ítems que hayan obtenido en idoneidad las puntuaciones más altas en el coeficiente de Aiken.

3) Procurar que los ítems finalmente seleccionados tengan puntuaciones altas en claridad. ${ }^{13}$

4) Valorar la facilidad de obtener la información que dota de contenido al ítem.

5) Revisar de nuevo la redundancia de cada ítem.

6) Seguir procurando una cierta proporción entre reglas y prácticas dentro de cada cesta y en el instrumento en general.

7) Asegurar que ninguna cesta queda con un número de ítems, entre reglas y prácticas, inferior a tres.

8) La diferencia en número de ítems entre las cestas no debe ser marcada.

Tras varias reuniones de todo el equipo investigador, en las que se adoptó un procedimiento pautado de verificación de todos estos requisitos, se logró en febrero de 2017 un acuerdo final sobre el instrumento RIMES que queda constituido por 39 ítems, de ellos 26 reglas y 13 prácticas, oscilando el número de ítems de cada cesta entre 4 y 5 (véase Tabla 5).

${ }^{13}$ Todavía se hacen algunas leves modificaciones de la redacción de algunos ítems. 
Tabla 5. Puntuación definitiva de los ítems seleccionados

\begin{tabular}{|c|c|c|c|}
\hline $\begin{array}{c}\text { Intervalos del coeficiente V de } \\
\text { Aiken }\end{array}$ & Claridad & Idoneidad & $\begin{array}{c}\text { Adecuación por } \\
\text { cestas }\end{array}$ \\
\hline 0.80 y más & 31 & 30 & 38 \\
\hline $0.75-0.79$ & 7 & 8 & 1 \\
\hline $0.70-0.74$ & 1 & 1 & 0 \\
\hline $0.63-0.69$ & 0 & 0 & 0 \\
\hline Not validated & 0 & 0 & 0 \\
\hline TOTAL & 39 & 39 & 39 \\
\hline
\end{tabular}

La lista de ítems finalmente seleccionados, con las puntuaciones en claridad, idoneidad y adecuación obtenidas por cada uno en el segundo pase de validación, es la siguiente (véase Tabla 6):

Tabla 6. Validación según V de Aiken

\begin{tabular}{|c|c|c|c|c|c|}
\hline $\mathrm{RoP}$ & Cesta & Lista de indicadores & Claridad & Idoneidad & $\begin{array}{l}\text { Adecuación por } \\
\text { cestas }\end{array}$ \\
\hline $\mathrm{R}$ & 7 & $\begin{array}{l}\text { 93. Nationals sentenced to imprisonment for } \\
\text { any criminal offence are not eligible for } \\
\text { welfare benefits for a certain period after } \\
\text { having served their sentences. }\end{array}$ & 0.92 & 0.96 & 0.96 \\
\hline $\mathrm{R}$ & 7 & $\begin{array}{l}\text { 92. Those sentenced to up to } 3 \text { years of } \\
\text { imprisonment for any criminal offence are not } \\
\text { eligible for public housing for a certain period } \\
\text { after having served their sentences. }\end{array}$ & 0.96 & 0.96 & 0.98 \\
\hline $\mathrm{R}$ & 3 & $\begin{array}{l}\text { 36. Default imprisonment is the sole } \\
\text { alternative to non-payment of a fine. }\end{array}$ & 0.94 & 0.94 & 0.94 \\
\hline $\mathrm{P}$ & 8 & $\begin{array}{l}\text { 107. The media regularly disclose the full } \\
\text { names, current addresses or pictures of ex- } \\
\text { felons. }\end{array}$ & 0.98 & 0.94 & 0.94 \\
\hline $\mathrm{R}$ & 9 & $\begin{array}{l}\text { 117. Youth justice provides custodial } \\
\text { sanctions of over } 10 \text { years. }\end{array}$ & 0.90 & 0.94 & 0.94 \\
\hline $\mathrm{R}$ & 7 & $\begin{array}{l}\text { 86. Those sentenced to up } 3 \text { years of } \\
\text { imprisonment for any criminal offence may be } \\
\text { deprived of the right to vote for over } 4 \text { years } \\
\text { after serving their sentences. }\end{array}$ & 0.88 & 0.92 & 0.92 \\
\hline $\mathrm{R}$ & 7 & $\begin{array}{l}\text { 88. Legally resident foreigners may be } \\
\text { deported if they receive a custodial sentence } \\
\text { up to one year or a non-custodial sentence. }\end{array}$ & 0.85 & 0.92 & 0.88 \\
\hline $\mathrm{R}$ & 8 & $\begin{array}{l}\text { 101. The criminal records of any citizen are } \\
\text { legally accessible through internet. }\end{array}$ & 1 & 0.92 & 0.98 \\
\hline$P$ & 8 & $\begin{array}{l}\text { 110. Most companies with over } 100 \\
\text { employees require a clean criminal record for } \\
\text { recruitment. }\end{array}$ & 0.87 & 0.90 & 0.94 \\
\hline$P$ & 9 & $\begin{array}{l}\text { 126. Alien minors are deported because of an } \\
\text { offence. }\end{array}$ & 0.85 & 0.90 & 0.90 \\
\hline $\mathrm{R}$ & 7 & $\begin{array}{l}\text { 91. Those sentenced to up to } 3 \text { years of } \\
\text { imprisonment are prohibited from doing } \\
\text { certain jobs not connected with their offences }\end{array}$ & 0.71 & 0.88 & 0.87 \\
\hline
\end{tabular}




\begin{tabular}{|c|c|c|c|c|c|}
\hline & & $\begin{array}{l}\text { or with law enforcement for a period of more } \\
\text { than } 5 \text { years their sentence has been } \\
\text { completed. }\end{array}$ & & & \\
\hline R & 1 & $\begin{array}{l}\text { 1. Any person may be arrested for repeated } \\
\text { street begging. }\end{array}$ & 0.94 & 0.88 & 0.96 \\
\hline $\mathrm{R}$ & 1 & $\begin{array}{l}\text { 3. At its discretion, the police may enforce } \\
\text { restrictions on specific individuals to access } \\
\text { some public spaces (parks, squares, streets...). }\end{array}$ & 0.85 & 0.88 & 0.92 \\
\hline $\mathrm{R}$ & 4 & $\begin{array}{l}\text { 43. Life imprisonment without release is } \\
\text { legally available. }\end{array}$ & 0.87 & 0.87 & 0.98 \\
\hline $\mathrm{P}$ & 4 & $\begin{array}{l}\text { 52. Life imprisonment is imposed on ethnical } \\
\text { or racial minorities, or on people in poverty in } \\
\text { over } 80 \% \text { of cases. }\end{array}$ & 0.87 & 0.87 & 0.85 \\
\hline $\mathrm{R}$ & 9 & $\begin{array}{l}\text { 120. 120. Minors' criminal records keep legal } \\
\text { effects after reaching the age of majority }\end{array}$ & 0.87 & 0.87 & 0.88 \\
\hline $\mathrm{P}$ & 1 & $\begin{array}{l}\text { 11. Discriminatory street police interventions } \\
\text { (stop and search, arrests, frisks/body } \\
\text { searches...) targeting specific groups occur } \\
\text { regularly. }\end{array}$ & 0.88 & 0.85 & 0.92 \\
\hline $\mathrm{R}$ & 6 & $\begin{array}{l}\text { 79. The maximum statutory term for pretrial } \\
\text { detention exceeds } 3 \text { years. }\end{array}$ & 0.90 & 0.85 & 0.85 \\
\hline $\mathrm{R}$ & 9 & $\begin{array}{l}113 \text {. Youth justice applies to children who are } \\
12 \text { years old or younger. }\end{array}$ & 0,85 & 0.85 & 0.90 \\
\hline $\mathrm{R}$ & 3 & $\begin{array}{l}\text { 33. In the case of prison sentences, neither } \\
\text { probation as an alternative to sentencing nor } \\
\text { suspended sentences are envisaged in the law. }\end{array}$ & 0.77 & 0.83 & 0.96 \\
\hline $\mathrm{P}$ & 3 & $\begin{array}{l}\text { 38. At least three quarters of the inmates are } \\
\text { serving their sentences in closed prisons. }\end{array}$ & 0.88 & 0.83 & 0.92 \\
\hline $\mathrm{R}$ & 4 & 42. Death penalty is legally available. & 1.00 & 0.83 & 0.94 \\
\hline $\mathrm{R}$ & 1 & 2. An individual may be arrested for loitering. & 0.79 & 0.83 & 0.92 \\
\hline $\mathrm{R}$ & 8 & $\begin{array}{l}\text { 100. Anyone may request information about } \\
\text { other people's criminal records without } \\
\text { needing to argue grounds established by law. }\end{array}$ & 0.81 & 0.83 & 0.94 \\
\hline $\mathrm{R}$ & 2 & $\begin{array}{l}\text { 21. The criminal justice system lacks indigent } \\
\text { defense services. }\end{array}$ & 0.83 & 0.81 & 0.92 \\
\hline $\mathrm{P}$ & 4 & $\begin{array}{l}\text { 50. Those sentenced to life imprisonment } \\
\text { regularly serve more than } 25 \text { years. }\end{array}$ & 0.96 & 0.81 & 0.88 \\
\hline $\mathrm{R}$ & 5 & $\begin{array}{l}55 \text {. The system lacks a specific prison regime } \\
\text { for young adults. }\end{array}$ & 0.96 & 0.81 & 0.90 \\
\hline $\mathrm{P}$ & 5 & $\begin{array}{l}\text { 68. Family and intimate visits take place at } \\
\text { intervals of over one month. }\end{array}$ & 0.98 & 0.81 & 0.92 \\
\hline $\mathrm{R}$ & 3 & $\begin{array}{l}\text { 35. The law lacks provisions for penalties } \\
\text { other than prison (community service, fines, } \\
\text { house arrest...) in cases of less serious } \\
\text { felonies. }\end{array}$ & 0.83 & 0.81 & 0.92 \\
\hline $\mathrm{R}$ & 5 & $\begin{array}{l}62 . \text { The law lacks statutory provisions } \\
\text { regulating inmates' legal assistance for } \\
\text { penitentiary matters. }\end{array}$ & 0.79 & 0.81 & 0.88 \\
\hline $\mathrm{P}$ & 9 & $\begin{array}{l}\text { 121. Custodial sanction is one out of three } \\
\text { most common sanctions applied to minors. }\end{array}$ & 0.83 & 0.79 & 0.90 \\
\hline $\mathrm{R}$ & 5 & $\begin{array}{l}\text { 64. The law requires payment of fees by the } \\
\text { inmate before claiming judicial review of } \\
\text { penitentiary decisions. }\end{array}$ & 0.77 & 0.79 & 0.88 \\
\hline $\mathrm{R}$ & 6 & 74. Preventive detention may last for an & 0.83 & 0.79 & 0.94 \\
\hline
\end{tabular}




\begin{tabular}{|c|c|c|c|c|c|}
\hline & & unlimited period of time. & & & \\
\hline $\mathrm{P}$ & 6 & $\begin{array}{l}\text { 82. Over } 30 \% \text { of the prison population is in } \\
\text { pre-trial detention. }\end{array}$ & 0.96 & 0.77 & 0.85 \\
\hline $\mathrm{R}$ & 2 & $\begin{array}{l}\text { 22. Payment of court fees is legally required } \\
\text { from the defendant in order to get access to } \\
\text { appellate review }\end{array}$ & 0.79 & 0.77 & 0.87 \\
\hline $\mathrm{P}$ & 2 & $\begin{array}{l}\text { 25. A significant number of mentally ill } \\
\text { inmates serve their sentences in regular } \\
\text { correctional facilities. }\end{array}$ & 0.85 & 0.77 & 0.75 \\
\hline $\mathrm{P}$ & 3 & $\begin{array}{l}\text { 37. The incarceration rate is higher than } 120 \\
\text { inmates per } 100,000 \text { inhabitants. }\end{array}$ & 0.87 & 0.77 & 0.85 \\
\hline $\mathrm{P}$ & 6 & $\begin{array}{l}\text { 81. The average length of preventive detention } \\
\text { exceeds } 5 \text { years. }\end{array}$ & 0.77 & 0.77 & 0.87 \\
\hline $\mathrm{R}$ & 2 & $\begin{array}{l}\text { 24. The regular term for police detention } \\
\text { established by the law exceeds } 5 \text { days. }\end{array}$ & 0.79 & 0.73 & 0.90 \\
\hline
\end{tabular}

A continuación, se formula el instrumento definitivo, estructurado por cestas (véase Tabla 7):

Tabla 7: Instrumento definitivo según cestas

\begin{tabular}{|c|c|c|}
\hline Cestas & Reglas & Prácticas \\
\hline \multirow[t]{3}{*}{$\begin{array}{l}\text { 1. Control of public } \\
\text { spaces }\end{array}$} & $\begin{array}{l}\text { 1. Any person may be arrested for } \\
\text { repeated street begging }\end{array}$ & \multirow{3}{*}{$\begin{array}{l}\text { 11. Discriminatory street police } \\
\text { interventions (stop and search, } \\
\text { arrests, frisks/body searches...) } \\
\text { targeting specific groups occur } \\
\text { regularly }\end{array}$} \\
\hline & $\begin{array}{l}\text { 2. An individual may be arrested for } \\
\text { loitering }\end{array}$ & \\
\hline & $\begin{array}{l}\text { 3. At its discretion, the police may } \\
\text { enforce restrictions on specific } \\
\text { individuals to access some public } \\
\text { spaces (parks, squares, streets...) }\end{array}$ & \\
\hline \multirow[t]{3}{*}{ 2. Legal safeguards } & $\begin{array}{l}\text { 21. The criminal justice system lacks } \\
\text { indigent defense services }\end{array}$ & \multirow{3}{*}{$\begin{array}{l}\text { 25. A significant number of } \\
\text { mentally ill inmates serve their } \\
\text { sentences in regular correctional } \\
\text { facilities }\end{array}$} \\
\hline & $\begin{array}{l}\text { 22. Payment of court fees is legally } \\
\text { required from the defendant in order to } \\
\text { get access to appellate review }\end{array}$ & \\
\hline & $\begin{array}{l}\text { 24. The regular term for police } \\
\text { detention established by the law } \\
\text { exceeds } 5 \text { days }\end{array}$ & \\
\hline \multirow[t]{3}{*}{$\begin{array}{l}\text { 3. Sentencing and } \\
\text { sanctions systems }\end{array}$} & $\begin{array}{l}\text { 33. In the case of prison sentences, } \\
\text { neither probation as an alternative to } \\
\text { sentencing nor suspended sentences are } \\
\text { envisaged in the law }\end{array}$ & $\begin{array}{l}37 . \text { The incarceration rate is } \\
\text { higher than } 120 \text { inmates per } \\
100,000 \text { inhabitants }\end{array}$ \\
\hline & $\begin{array}{l}\text { 35. The law lacks provisions for } \\
\text { penalties other than prison (community } \\
\text { service, fines, house arrest...) in case } \\
\text { of less serious felonies }\end{array}$ & \multirow[t]{2}{*}{$\begin{array}{l}\text { 38. At least three quarters of the } \\
\text { inmates are serving their } \\
\text { sentences in closed prisons }\end{array}$} \\
\hline & $\begin{array}{l}\text { 36. Default imprisonment is the sole } \\
\text { alternative to non-payment of a fine }\end{array}$ & \\
\hline \multirow[t]{2}{*}{ 4. Harshest penalties } & 42. Death penalty is legally available & $\begin{array}{l}\text { 50. Those sentenced to life } \\
\text { imprisonment regularly serve } \\
\text { more than } 25 \text { years }\end{array}$ \\
\hline & $\begin{array}{l}\text { 43. Life imprisonment without release } \\
\text { is legally available }\end{array}$ & $\begin{array}{l}\text { 52. In over } 80 \% \text { of life sentences } \\
\text { those serving time are from and } \\
\text { ethnic minority background or } \\
\text { were living in poverty }\end{array}$ \\
\hline
\end{tabular}




\begin{tabular}{|c|c|c|}
\hline \multirow[t]{3}{*}{ 5. Prison rules } & $\begin{array}{l}\text { 55. The system lacks a specific prison } \\
\text { regime for young adults }\end{array}$ & \multirow{3}{*}{$\begin{array}{l}\text { 68. Family and intimate visits } \\
\text { take place al intervals of over } \\
\text { one month }\end{array}$} \\
\hline & $\begin{array}{l}\text { 62. The law lacks statutory provisions } \\
\text { regulating inmates' legal assistance for } \\
\text { penitentiary matters }\end{array}$ & \\
\hline & $\begin{array}{l}\text { 64. The law requires payment of fees } \\
\text { by the inmate before claiming judicial } \\
\text { review of penitentiary decisions }\end{array}$ & \\
\hline \multirow[t]{2}{*}{$\begin{array}{l}\text { 6. Preventive } \\
\text { intervention }\end{array}$} & $\begin{array}{l}\text { 74. Preventive detention may last for } \\
\text { an unlimited period of time }\end{array}$ & $\begin{array}{l}\text { 81. The average length of } \\
\text { preventive detention exceeds } 5 \\
\text { years }\end{array}$ \\
\hline & $\begin{array}{l}\text { 79. The maximum statutory term for } \\
\text { pretrial detention exceeds } 3 \text { years }\end{array}$ & $\begin{array}{l}\text { 82. Over } 30 \% \text { of the prison } \\
\text { population is in pre-trial } \\
\text { detention }\end{array}$ \\
\hline \multirow[t]{5}{*}{$\begin{array}{l}\text { 7. Legal and social status } \\
\text { of offenders and ex- } \\
\text { offenders }\end{array}$} & $\begin{array}{l}\text { 86. Those sentenced to up } 3 \text { years of } \\
\text { imprisonment for any criminal offence } \\
\text { may be deprived of the right to vote for } \\
\text { over } 4 \text { years after serving their } \\
\text { sentences }\end{array}$ & \\
\hline & $\begin{array}{l}\text { 88. Legally resident foreigners may be } \\
\text { deported if they receive a custodial } \\
\text { sentence up to one year or a } \\
\text { noncustodial sentence }\end{array}$ & \\
\hline & $\begin{array}{l}\text { 91. Those sentenced to up to } 3 \text { years of } \\
\text { imprisonment are prohibited from } \\
\text { doing certain jobs not connected with } \\
\text { their offences or with law enforcement } \\
\text { for a period of more than } 5 \text { years their } \\
\text { sentence has been completed }\end{array}$ & \\
\hline & $\begin{array}{l}\text { 92. Those sentenced to up to } 3 \text { years of } \\
\text { imprisonment for any criminal offence } \\
\text { are not eligible for public housing for a } \\
\text { certain period after having served their } \\
\text { sentences }\end{array}$ & \\
\hline & $\begin{array}{l}\text { 93. Nationals sentenced to } \\
\text { imprisonment for any criminal offence } \\
\text { are not eligible for welfare benefits for } \\
\text { a certain period after having served } \\
\text { their sentences }\end{array}$ & \\
\hline \multirow[t]{2}{*}{$\begin{array}{l}\text { 8. Police and criminal } \\
\text { records }\end{array}$} & $\begin{array}{l}\text { 100. Anyone may request information } \\
\text { about other people's criminal records } \\
\text { without needing to argue grounds } \\
\text { established by law }\end{array}$ & $\begin{array}{l}\text { 107. The media regularly } \\
\text { disclose the full names, current } \\
\text { addresses or pictures of ex felons }\end{array}$ \\
\hline & $\begin{array}{l}\text { 101. The criminal records of any } \\
\text { citizen are legally accessible through } \\
\text { internet }\end{array}$ & $\begin{array}{l}\text { 110. Most companies with over } \\
100 \text { employees require a clean } \\
\text { criminal record for recruitment }\end{array}$ \\
\hline \multirow[t]{3}{*}{ 9. Youth criminal justice } & $\begin{array}{l}\text { 113. Youth justice applies to children } \\
\text { who are } 12 \text { years old or younger }\end{array}$ & $\begin{array}{l}\text { 121. Custodial sanction is one } \\
\text { out of three most common } \\
\text { sanctions applied to minors }\end{array}$ \\
\hline & $\begin{array}{l}\text { 117. Youth justice provides custodial } \\
\text { sanctions of over } 10 \text { years }\end{array}$ & \multirow[t]{2}{*}{$\begin{array}{l}\text { 126. Alien minors are deported } \\
\text { because of an offence }\end{array}$} \\
\hline & $\begin{array}{l}\text { 120. Minors' criminal records keep } \\
\text { legal effects after reaching the age of } \\
\text { majority }\end{array}$ & \\
\hline
\end{tabular}




\section{Polít. Crim. Vol. 15, N 30 (Diciembre 2020), Art. 6, pp. 670-693 \\ [http://politcrim.com/wp-content/uploads/2020/12/Vol15N30A6.pdf]}

Una vez disponible el instrumento de medida RIMES, el equipo de investigación procedió a su aplicación en España, como proyecto piloto, antes de hacerlo en otros países. La experiencia de su aplicación en España y sus resultados se expondrán en otro estudio.

\section{Conclusiones}

Esta investigación ha partido del presupuesto de que el saber político-criminal precisa de instrumentos metodológicos rigurosos que le permitan evaluar y proponer políticas de control penal correctas y eficaces.

Para ello es necesario que se escoja un criterio de análisis de la política criminal contemporánea capaz de ofrecer resultados significativos, comprensivos y consistentes. En esta investigación se ha escogido, en contraste con lo que suele ser habitual en la reflexión político-criminal actual, la dimensión de la exclusión social que el control penal es susceptible de producir en colectivos especialmente afectados por la intervención punitiva.

No basta con elegir un criterio analítico prometedor, sino que este se ha de hacer operativo, para que pueda desarrollar todas sus potencialidades explicativas y críticas. La herramienta RIMES de comparación de las políticas criminales nacionales desde la perspectiva de la exclusión social que producen sobre colectivos especialmente sensibles, cuya creación se explica en este artículo, constituye una realización de ese objetivo.

El citado instrumento, que mide la presencia en un determinado sistema nacional de control penal de reglas y prácticas punitivas socialmente excluyentes, ha sido validado de un modo metodológicamente riguroso a través de una doble valoración interjueces en la que han participado en torno a un centenar de expertos pertenecientes a 18 países del mundo occidental desarrollado.

Su aplicación a un amplio número de países occidentales desarrollados permitirá comparar sus respectivas políticas criminales nacionales a partir de una dimensión especialmente significativa de las prestaciones de sus sistemas de control penal. Se podrá trazar un continuo, dentro de una escala, entre políticas criminales nacionales más o menos socialmente excluyentes, identificar en qué medida se están expandiendo técnicas de intervención excluyentes en el mundo occidental desarrollado, y agrupar esos resultados alrededor de nueve ámbitos relevantes de la intervención penal.

En último término, suministrará información de gran relevancia a la hora de promover y diseñar políticas criminales socialmente menos excluyentes de los colectivos más afectados por la intervención penal. 


\section{Bibliografía citada}

AIKEN, Lewis R. (1980): "Content validity and reliability of single items or questionnaires", en: Educational and Psychological Measurement (40). pp. 955-959.

BALVIG, Flemming (2004): "When law and order returned to Denmark", en: Journal of Scandinavian Studies in Criminology and Crime Prevention. (Vol. 5). pp. 167-187.

BRODEUR, Jean Paul (2007): "Comparative penology in perspective", en: TONRY, Michael (Ed.), Crime, Punishment and Politics in Comparative Perspective. (Chicago: University of Chicago Press). pp. 49-91.

CABERO ALMENARA, Julio; BARROSO OSUNA, Julio (2013): La utilización del juicio de experto para la evaluación de TIC: El coeficiente de competencia experta, en: Bordón $(65,2)$. pp. 25-38.

CAVADINO, Mick; DIGNAN, James (2006): Penal Systems (London: Sage Publications). pp. 380.

CRUZ RAMÍREZ, Miguel (2009): El método Delphi en las investigaciones educacionales (Editorial Academia).

DÍEZ-RIPOLLÉS, José Luis (2011): “La dimensión inclusión / exclusión social como guía de la política criminal comparada", en: Revista electrónica de ciencia penal y criminología ( $\left.\mathrm{N}^{\circ} \mathrm{v} 13-12\right), \quad$ pp. 1-36. Disponible en http://criminet.ugr.es/recpc/13/recpc13-12.pdf [Visitado 17/12/20210]

DÍEZ-RIPOLLÉS, José Luis (2013): "Social Inclusion and Comparative Criminal Justice Policy", en: Journal of Scandinavian Studies in Criminology and Crime Prevention (14), pp. 62-78.

DOWNES, David; HANSEN, Karen (2006): "Welfare and punishment in comparative perspective", en: Armstrong Sarah; McAra Leslie (Ed.), Perspectives on Punishment. (Oxford: Oxford University Press).

ESCOBAR-PÉREZ, Jazmine; CUERVO-MARTÍNEZ, Ángela, (2008): "Validez de contenido y juicio de expertos: Una aproximación a su utilización", en: Avances en medición, $\left(\mathrm{N}^{\circ} 6\right)$. pp. 27-36.

HINDS, Lyn (2005): “Crime control in Western countries”, en: PRATT, John; BROWN, David; BROWN, Mark; HALLSWORTH, Simón; MORRISON, Wayne, The New Punitiveness. (Cullompton: Willan Publishing). pp. 346.

LAPPI-SEPPÄLÄ, Tapio (2008): “Trust, Welfare, and Political Culture”, en: TONRY, Michael (Ed.) Crime and Justice. (Chicago: University of Chicago Press). pp. 313387.

LARRAURI PIJOAN, Elena (2009): "La economía política del castigo", en: Revista electrónica de ciencia penal y criminología (11-06). pp. 1-22.

NELKEN, David (2010): Comparative Criminal Justice (Los Ángeles: Sage Publications). Pp.136.

PRATT, John (2008): "Scandinavian exceptionalism in an era of penal excess I", en: British Journal of Criminology (48).

REGUANT-ÁLVAREZ, Mercedes; TORRADO-FONSECA, Mercedes (2016): El método Delphi, en: REIRE, Revista d'Innovació i Recerca en Educació, (9,1). Pp. 87-102.

ROCHÉ, Sebastian (2007): "Criminal justice policy in France”, en: TONRY, Michael (Ed.), Crime, Punishment and Politics in Comparative Perspective. (Chicago: University of Chicago Press). 
SNACKEN, Sonja (2007): "Penal policy and practice in Belgium", en: TONRY, Michael (Ed.), Punishment and Politics in Comparative Perspective. (Chicago: University of Chicago Press).

TONRY, Michel (2007): "Determinants of penal policies", en: TONRY, Michael (Ed.) Crime, Punishment and Politics in Comparative Perspective. (Chicago: University of Chicago Press).

WEBSTER, Cheryl; DOOB, Anthony (2007): "Punitive trends and stable imprisonment rates in Canada, en: TONRY, Michael (Ed.), Crime, Punishment and Politics in Comparative Perspective. (Chicago: University of Chicago Press).

ZAFFARONI, Raúl, (2007): El enemigo en el derecho penal (México DF: Ediciones Coyoacán). 